\title{
Strong Linear Dependence and Unbiased Distribution of Non-propagative Vectors
}

\author{
Yuliang Zheng $^{1}$ and Xian-Mo Zhang $^{2}$ \\ 1 School of Comp \& Info Tech, Monash University \\ McMahons Road, Frankston, Melbourne, VIC 3199, Australia \\ yuliang@pscit.monash.edu.au \\ http://www.pscit.monash.edu.au/links/ \\ 2 School of Info Tech \& Comp Sci \\ The University of Wollongong, Wollongong \\ NSW 2522, Australia \\ xianmo@cs.uow.edu.au
}

\begin{abstract}
This paper proves (i) in any $(n-1)$-dimensional linear subspace, the non-propagative vectors of a function with $n$ variables are linearly dependent, (ii) for this function, there exists a non-propagative vector in any $(n-2)$-dimensional linear subspace and there exist three non-propagative vectors in any $(n-1)$-dimensional linear subspace, except for those functions whose nonlinearity takes special values.

Keywords: Cryptography, Boolean Function, Propagation, Nonlinearity.
\end{abstract}

\section{Introduction}

In examining the nonlinearity properties of a function $f$ with $n$ variables, it is important to understand $\Re_{f}$, the set of so-called non-propagative vectors where $f$ does not satisfy the propagation criterion. In this work, we are concerned with both $\# \Re_{f}$ (the number of non-propagative vectors in $\Re_{f}$ ) and the distribution of $\Re_{f}$. More specifically, we prove two properties of $\Re$. One is called the strong linear dependence and the other the unbiased distribution, of $\Re$.

The strong linear dependence property states that if $W$ is a $(n-1)$-dimensional linear subspace satisfying $\#(\Re \cap W) \geq 4$, then the non-zero vectors in $\Re \cap W$ are linearly dependent. This improves a previously known result. The unbiased distribution property says that any function $f$ with $n$ variables, except for those whose nonlinearity takes the special value of $2^{n-1}-2^{\frac{1}{2}(n-1)}, 2^{n-1}-2^{\frac{1}{2} n}$ or $2^{n-1}-2^{\frac{1}{2} n-1}$, fulfills the condition that every $(n-2)$-dimensional linear subspace contains a non-zero vector in $\Re_{f}$ and every $(n-1)$-dimensional linear subspace contains at least three non-zero vectors in $\Re_{f}$. In special cases, \#( $\cap$ $W$ ) may significantly effect other cryptographic properties of a function. The strong linear dependence and the unbiased distribution are helpful for the design of cryptographic functions as these conclusions provide more information on the number and the status of non-propagative vectors in any $(n-1)$-dimensional linear subspace. 


\section{Cryptographic Criteria of Boolean Functions}

We consider functions from $V_{n}$ to $G F(2)$ (or simply functions on $V_{n}$ ), $V_{n}$ is the vector space of $n$ tuples of elements from $G F(2)$. The truth table of a function $f$ on $V_{n}$ is a $(0,1)$-sequence defined by $\left(f\left(\alpha_{0}\right), f\left(\alpha_{1}\right), \ldots, f\left(\alpha_{2^{n}-1}\right)\right)$, and the sequence of $f$ is a $(1,-1)$-sequence defined by $\left((-1)^{f\left(\alpha_{0}\right)},(-1)^{f\left(\alpha_{1}\right)}\right.$, $\left.\ldots,(-1)^{f\left(\alpha_{2^{n}-1}\right)}\right)$, where $\alpha_{0}=(0, \ldots, 0,0), \alpha_{1}=(0, \ldots, 0,1), \ldots, \alpha_{2^{n-1}-1}=$ $(1, \ldots, 1,1)$. The matrix of $f$ is a $(1,-1)$-matrix of order $2^{n}$ defined by $M=$ $\left((-1)^{f\left(\alpha_{i} \oplus \alpha_{j}\right)}\right)$ where $\oplus$ denotes the addition in $G F(2) . f$ is said to be balanced if its truth table contains an equal number of ones and zeros.

Given two sequences $\tilde{a}=\left(a_{1}, \cdots, a_{m}\right)$ and $\tilde{b}=\left(b_{1}, \cdots, b_{m}\right)$, their componentwise product is defined by $\tilde{a} * \tilde{b}=\left(a_{1} b_{1}, \cdots, a_{m} b_{m}\right)$. In particular, if $m=2^{n}$ and $\tilde{a}, \tilde{b}$ are the sequences of functions $f$ and $g$ on $V_{n}$ respectively, then $\tilde{a} * \tilde{b}$ is the sequence of $f \oplus g$ where $\oplus$ denotes the addition in $G F(2)$.

Let $\tilde{a}=\left(a_{1}, \cdots, a_{m}\right)$ and $\tilde{b}=\left(b_{1}, \cdots, b_{m}\right)$ be two sequences or vectors, the scalar product of $\tilde{a}$ and $\tilde{b}$, denoted by $\langle\tilde{a}, \tilde{b}\rangle$, is defined as the sum of the component-wise multiplications. In particular, when $\tilde{a}$ and $\tilde{b}$ are from $V_{m},\langle\tilde{a}, \tilde{b}\rangle=$ $a_{1} b_{1} \oplus \cdots \oplus a_{m} b_{m}$, where the addition and multiplication are over $G F(2)$, and when $\tilde{a}$ and $\tilde{b}$ are $(1,-1)$-sequences, $\langle\tilde{a}, \tilde{b}\rangle=\sum_{i=1}^{m} a_{i} b_{i}$, where the addition and multiplication are over the reals.

An affine function $f$ on $V_{n}$ is a function that takes the form of $f\left(x_{1}, \ldots, x_{n}\right)=$ $a_{1} x_{1} \oplus \cdots \oplus a_{n} x_{n} \oplus c$, where $a_{j}, c \in G F(2), j=1,2, \ldots, n$. Furthermore $f$ is called a linear function if $c=0$.

A $(1,-1)$-matrix $N$ of order $n$ is called a Hadamard matrix if $N N^{T}=n I_{n}$, where $N^{T}$ is the transpose of $N$ and $I_{n}$ is the identity matrix of order $n$. A Sylvester-Hadamard matrix of order $2^{n}$, denoted by $H_{n}$, is generated by the following recursive relation

$$
H_{0}=1, H_{n}=\left[\begin{array}{cc}
H_{n-1} & H_{n-1} \\
H_{n-1} & -H_{n-1}
\end{array}\right], n=1,2, \ldots
$$

Let $\ell_{i}, 0 \leq i \leq 2^{n}-1$, be the $i$ row of $H_{n}$. It is known that $\ell_{i}$ is the sequence of a linear function $\varphi_{i}(x)$ defined by the scalar product $\varphi_{i}(x)=\left\langle\alpha_{i}, x\right\rangle$, where $\alpha_{i}$ is the $i$ th vector in $V_{n}$ according to the ascending alphabetical order.

The Hamming weight of a $(0,1)$-sequence $\xi$, denoted by $W(\xi)$, is the number of ones in the sequence. Given two functions $f$ and $g$ on $V_{n}$, the Hamming distance $d(f, g)$ between them is defined as the Hamming weight of the truth table of $f(x) \oplus g(x)$, where $x=\left(x_{1}, \ldots, x_{n}\right)$.

Definition 1. The nonlinearity of a function $f$ on $V_{n}$, denoted by $N_{f}$, is the minimal Hamming distance between $f$ and all affine functions on $V_{n}$, i.e., $N_{f}=$ $\min _{i=1,2, \ldots, 2^{n+1}} d\left(f, \varphi_{i}\right)$ where $\varphi_{1}, \varphi_{2}, \ldots, \varphi_{2^{n+1}}$ are all the affine functions on $V_{n}$.

The following characterisations of nonlinearity will be useful (for a proof see for instance 2 ). 
Lemma 1. The nonlinearity of $f$ on $V_{n}$ can be expressed by

$$
N_{f}=2^{n-1}-\frac{1}{2} \max \left\{\left|\left\langle\xi, \ell_{i}\right\rangle\right|, 0 \leq i \leq 2^{n}-1\right\}
$$

where $\xi$ is the sequence of $f$ and $\ell_{0}, \ldots, \ell_{2^{n}-1}$ are the rows of $H_{n}$, namely, the sequences of linear functions on $V_{n}$.

Definition 2. Let $f$ be a function on $V_{n}$. For a vector $\alpha \in V_{n}$, denote by $\xi(\alpha)$ the sequence of $f(x \oplus \alpha)$. Thus $\xi(0)$ is the sequence of $f$ itself and $\xi(0) * \xi(\alpha)$ is the sequence of $f(x) \oplus f(x \oplus \alpha)$. Set

$$
\Delta_{f}(\alpha)=\langle\xi(0), \xi(\alpha)\rangle
$$

the scalar product of $\xi(0)$ and $\xi(\alpha) . \Delta(\alpha)$ is called the auto-correlation of $f$ with a shift $\alpha$. Write

$$
\Delta_{M}=\max \left\{|\Delta(\alpha)| \mid \alpha \in V_{n}, \alpha \neq 0\right\}
$$

We omit the subscript of $\Delta_{f}(\alpha)$ if no confusion occurs.

Definition 3. Let $f$ be a function on $V_{n}$. We say that $f$ satisfies the propagation criterion with respect to $\alpha$ if $f(x) \oplus f(x \oplus \alpha)$ is a balanced function, where $x=\left(x_{1}, \ldots, x_{n}\right)$ and $\alpha$ is a vector in $V_{n}$. Furthermore $f$ is said to satisfy the propagation criterion of degree $k$ if it satisfies the propagation criterion with respect to every non-zero vector $\alpha$ whose Hamming weight is not larger than $k$ (see $\left.{ }^{3} 7\right)$.

The strict avalanche criterion $(S A C), 5$ is the same as the propagation criterion of degree one.

Obviously, $\Delta(\alpha)=0$ if and only if $f(x) \oplus f(x \oplus \alpha)$ is balanced, i.e., $f$ satisfies the propagation criterion with respect to $\alpha$.

Definition 4. Let $f$ be a function on $V_{n} . \alpha \in V_{n}$ is called a linear structure of $f$ if $|\Delta(\alpha)|=2^{n}$ (i.e., $f(x) \oplus f(x \oplus \alpha)$ is a constant).

For any function $f, \Delta\left(\alpha_{0}\right)=2^{n}$, where $\alpha_{0}$ is the zero vector on $V_{n}$. It is easy to verify that the set of all linear structures of a function $f$ form a linear subspace of $V_{n}$, whose dimension is called the linearity of $f$. It is also well-known that if $f$ has non-zero linear structures, then there exists a nonsingular $n \times n$ matrix $B$ over $G F(2)$ such that $f(x B)=g(y) \oplus h(z)$, where $x=(y, z), y \in V_{p}$, $z \in V_{q}, g$ is a function on $V_{p}$ that has no non-zero linear structures, and $h$ is an affine function on $V_{q}$.

The following lemma is the re-statement of a relation proved in Section 2 of II.

Lemma 2. For every function $f$ on $V_{n}$, we have

$$
\left(\Delta\left(\alpha_{0}\right), \Delta\left(\alpha_{1}\right), \ldots, \Delta\left(\alpha_{2^{n}-1}\right)\right) H_{n}=\left(\left\langle\xi, \ell_{0}\right\rangle^{2},\left\langle\xi, \ell_{1}\right\rangle^{2}, \ldots,\left\langle\xi, \ell_{2^{n}-1}\right\rangle^{2}\right) .
$$

where $\xi$ denotes the sequence of $f$ and $\ell_{i}$ is the ith row of $H_{n}, i=0,1, \ldots, 2^{n}-1$.

The balance and the nonlinearity are necessary in most cases. The propagation or especially the SAC, is an important cryptographic criterion. 


\section{Introduction to $\Re$}

Notation 1. Let $f$ be a function on $V_{n}$. Set $\Re_{f}=\left\{\alpha \mid \Delta(\alpha) \neq 0, \alpha \in V_{n}\right\}$, $\Delta_{M}=\max \left\{\mid \Delta(\alpha) \| \alpha \in V_{n}, \alpha \neq 0\right\}$.

We simply write $\Re_{f}$ as $\Re$ if no confusion occurs. It is easy to verify that $\# \Re$ and $\Delta_{M}$ are invariant under any nonsingular linear transformation on the variables, where \# denotes the cardinal number of a set.

$\# \Re$ and the distribution of $\Re$ reflects the propagation characteristics, while $\Delta_{M}$ forecasts the avalanche property of the function. Therefore information on $\Re$ and $\Delta_{M}$ is useful in determining important cryptographic characteristics of $f$. Usually, small \# $\#$ and $\Delta_{M}$ are desirable.

Definition 5. A function $f$ on $V_{n}$ is called a bent function 4 if $\left\langle\xi, \ell_{i}\right\rangle^{2}=2^{n}$ for every $i=0,1, \ldots, 2^{n}-1$, where $\ell_{i}$ is the $i$ th row of $H_{n}$.

A bent function on $V_{n}$ exists only when $n$ is even, and it achieves the highest possible nonlinearity $2^{n-1}-2^{\frac{1}{2} n-1}$. The algebraic degree of bent functions on $V_{n}$ is at most $\frac{1}{2} n$. From 4 and Parseval's equation, we have the following:

Theorem 1. Let $f$ be a function on $V_{n}$. Then the following statements are equivalent: (i) $f$ is bent, (ii) $\# \Re=1$, (iii) $\Delta_{M}=0$, (iv) the nonlinearity of $f$, $N_{f}$, satisfies $N_{f}=2^{n-1}-2^{\frac{1}{2} n-1}$, (v) the matrix of $f$ is an Hadamard matrix.

The following result is called the linear dependence theorem that can be found in 7

Theorem 2. Let $f$ be a function on $V_{n}$ that satisfies the propagation criterion with respect to all but $k+1$ vectors $0, \beta_{1}, \ldots, \beta_{k}$ in $V_{n}$, where $k \geq 2$. Then $\beta_{1}, \ldots, \beta_{k}$ are linearly dependent, namely, there exist $k$ constants $c_{1}, \ldots, c_{k} \in$ $G F(2)$, not all of which are zeros, such that $c_{1} \beta_{1} \oplus \cdots \oplus c_{k} \beta_{k}=0$.

Note that $n+1$ non-zero vectors in $V_{n}$ must be linearly dependent. Hence if $\# \Re \geq n+2$ (i.e., $\#(\Re-\{0\}) \geq n+1)$ then Theorem $\boldsymbol{Z}$ is trivial. For this reason, we improve Theorem $\boldsymbol{Z}$ in this paper. We prove two properties of $\Re$ : the strong linear dependence and the unbiased distribution of $\Re$.

\section{The Strong Linear Dependence Theorem}

Note the $i$ th (i.e., the $\alpha_{i}$ th) row of $H_{n}$, where $\alpha_{i} \in V_{n}$ is the binary representation of integer $j, j=0,1, \ldots, 2^{n}-1$, is the sequence of linear function $\varphi_{i}(x)=\left\langle\alpha_{i}, x\right\rangle$. Lemma 4 of 7 can be restated as follows:

Lemma 3. Let $Q$ be the $2^{n} \times q$ that consists of the $\alpha_{j_{1}}$ th, $\ldots$, the $\alpha_{j_{q}}$ th rows of $H_{n}$, where each $\alpha_{j} \in V_{n}$ is the binary representation of integer $j, 0 \leq j \leq$ $2^{n}-1$. If $\alpha_{j_{1}}, \ldots, \alpha_{j_{q}}$ are linearly independent then each $\left(a_{1}, \ldots, a_{q}\right)^{T}$, where each $a_{j}= \pm 1$, appears as a column in $Q$ precisely $2^{n-q}$ times. 
The following Lemma can be found in 7 .

Lemma 4. Let $n \geq 3$ be a positive integer and $2^{n}=\sum_{j=1}^{4} a_{j}^{2}$ where $a_{1} \geq a_{2} \geq$ $a_{3} \geq a_{4} \geq 0$ and each $a_{j}$ is an integer. We have the following statements:

(i) if $n$ is add, then $a_{1}^{2}=a_{2}^{2}=2^{n-1}, a_{3}=a_{4}=0$,

(ii) if $n$ is even, then $a_{1}^{2}=2^{n}, a_{2}=a_{3}=a_{4}=0$ or $a_{1}^{2}=a_{2}^{2}=a_{3}^{2}=a_{4}^{2}=2^{n-2}$.

Lemma 5. For every function $f$ on $V_{n}$, we have

$$
\begin{aligned}
& 2\left(\Delta\left(\alpha_{0}\right), \Delta\left(\alpha_{2}\right), \ldots, \Delta\left(\alpha_{2^{n}-2}\right)\right) H_{n-1} \\
& =\left(\left\langle\xi, \ell_{0}\right\rangle^{2}+\left\langle\xi, \ell_{1}\right\rangle^{2},\left\langle\xi, \ell_{2}\right\rangle^{2}+\left\langle\xi, \ell_{3}\right\rangle^{2}, \ldots,\left\langle\xi, \ell_{2^{n}-2}\right\rangle^{2}+\left\langle\xi, \ell_{2^{n}-1}\right\rangle^{2}\right)
\end{aligned}
$$

where $\xi$ denotes the sequence of $f$ and $\ell_{i}$ is the ith row of $H_{n}, i=0,1, \ldots, 2^{n}-1$.

\section{Proof. From Lemma}

$$
2^{n}\left(\Delta\left(\alpha_{0}\right), \Delta\left(\alpha_{1}\right), \ldots, \Delta\left(\alpha_{2^{n}-1}\right)\right)=\left(\left\langle\xi, \ell_{0}\right\rangle^{2},\left\langle\xi, \ell_{1}\right\rangle^{2}, \ldots,\left\langle\xi, \ell_{2^{n}-1}\right\rangle^{2}\right) H_{n}
$$

Comparing the 0 th, the 2 nd, ..., the $\left(2^{n}-2\right)$ th terms in the two sides of equality

II, we obtain

$$
\begin{aligned}
& 2^{n}\left(\Delta\left(\alpha_{0}\right), \Delta\left(\alpha_{2}\right), \ldots, \Delta\left(\alpha_{2^{n}-2}\right)\right) \\
& =\left(\left\langle\xi, \ell_{0}\right\rangle^{2}+\left\langle\xi, \ell_{1}\right\rangle^{2},\left(\left\langle\xi, \ell_{2}\right\rangle^{2}+\left\langle\xi, \ell_{3}\right\rangle^{2}, \ldots,\left\langle\xi, \ell_{2^{n}-2}\right\rangle^{2}+\left\langle\xi, \ell_{2^{n}-1}\right\rangle^{2}\right) H_{n-1}\right.
\end{aligned}
$$

This proves the lemma.

The following theorem is called the strong linearly dependence theorem which is an improvement on Theorem 2 (the linearly dependence theorem).

Theorem 3. Let $f$ be a function on $V_{n}$, and $W$ be a $(n-1)$-dimensional linear subspace satisfying $\Re \cap W=\left\{0, \beta_{1}, \ldots, \beta_{k}\right\}(k \geq 3)$. Then $\beta_{1}, \ldots, \beta_{k}$ are linearly dependent, namely, there exist $k$ constants $c_{1}, \ldots, c_{k} \in G F(2)$ with $\left(c_{1}, \ldots, c_{k}\right) \neq$ $(0, \ldots, 0)$, such that $c_{1} \beta_{1} \oplus \cdots \oplus c_{k} \beta_{k}=0$.

Proof. The theorem is obviously true if $k>n$. Now we prove the theorem for $k \leq n$. We only need to prove the lemma in the special case when $W$ is composed of $\alpha_{0}, \alpha_{2}, \ldots, \alpha_{2^{n}-2}$, where $\alpha_{2 j} \in V_{n}$ is the binary representation of an even number $2 j, j=0,1, \ldots, 2^{n-1}-1$. In other words, $W$ is composed of all the vectors in $V_{n}$, that can be expressed in the form $\left(a_{1}, \ldots, a_{n-1}, 0\right)$, where each $a_{j} \in G F(2)$. In the general case, we can use a nonsingular linear transformation on the variables so as to change $W$ into the special case. Let $\xi$ be the sequence of $f$.

Since $\beta_{j} \in W, j=1, \ldots, k, \beta_{j}$ can be expressed as $\beta_{j}=\left(\gamma_{j}, 0\right)$ where $\gamma_{j} \in V_{n-1}, j=1, \ldots, k$, and $0 \in G F(2)$. 
Let $P$ be a $(k+1) \times 2^{n-1}$ matrix composed of the 0 th, the $\gamma_{1}$ th, .., the $\gamma_{k}$ th rows of $H_{n-1}$. Set $a_{j}^{2}=\left\langle\xi, \ell_{j}\right\rangle^{2}, j=0,1, \ldots, 2^{n}-1$. Note that $\Delta(\alpha)=0$ if $\alpha \notin\left\{0, \beta_{1}, \ldots, \beta_{k}\right\}$. Hence the equality in Lemma 5 can be specialized as

$$
2\left(\Delta(0), \Delta\left(\beta_{1}\right), \ldots, \Delta\left(\beta_{k}\right)\right) P=\left(a_{0}^{2}+a_{1}^{2}, a_{2}^{2}+a_{3}^{2}, \ldots, a_{2^{n}-2}^{2}+a_{2^{n}-1}^{2}\right)
$$

where $\Delta(0)$ is identical to $\Delta\left(\alpha_{0}\right)$ where $\alpha_{0}=0$.

Write $P=\left(p_{i j}\right), i=0,1, \ldots k, j=0,1, \ldots, 2^{n-1}-1$. As the top row of $P$ is $(1,1, \ldots, 1)$, from 2$)$

$$
2\left(\Delta(0)+\sum_{i=1}^{k} p_{i j} \Delta\left(\beta_{i}\right)\right)=a_{2 j}^{2}+a_{2 j+1}^{2}
$$

$j=0,1, \ldots, 2^{n-1}-1$. Let $P^{*}$ be the submatrix of $P$ obtained by removing the top row from $P$.

We now prove the theorem by contradiction. Suppose $k$ vectors in $V_{n}, \beta_{1}$, $\ldots, \beta_{k}$, are linearly independent. Hence $k$ vectors in $V_{n-1}, \gamma_{1}, \ldots, \gamma_{k}$, are also linearly independent and hence $k \leq n-1$.

Applying Lemma 3 to matrix $P^{*}$, we conclude that each $k$-dimensional $(1,-1)$-vector appears in $P^{*}$, as a column vector of $P^{*}$ precisely $2^{n-1-k}$ times. Thus for each fixed $j$ there exists a number $j_{0}, 0 \leq j_{0} \leq 2^{n-1}-1$, such that $\left(p_{1 j_{0}}, \ldots, p_{k j_{0}}\right)=-\left(p_{1 j}, \ldots, p_{k j}\right)$ and hence

$$
2\left(\Delta(0)-\sum_{i=1}^{k} p_{i j_{0}} \Delta\left(\beta_{i}\right)\right)=a_{j_{0}}^{2}+a_{2 j_{0}+1}^{2}
$$

Adding 3 and 4 together, we have $4 \Delta(0)=a_{j}^{2}+a_{2 j+1}^{2}+a_{j_{0}}^{2}+a_{2 j_{0}+1}^{2}$. Hence $a_{j}^{2}+a_{2 j+1}^{2}+a_{j_{0}}^{2}+a_{2 j_{0}+1}^{2}=2^{n+2}$. There are two cases to be considered: even $n$ and odd $n$.

Case 1: $n$ is odd. By using Lemma 4

$$
\left\{a_{j}^{2}, a_{2 j+1}^{2}, a_{j_{0}}^{2}, a_{2 j_{0}+1}^{2}\right\}=\left\{2^{n+1}, 2^{n+1}, 0,0\right\}, j=0,1, \ldots, 2^{n-1}
$$

Hence from 3 , we have $\Delta(0)+\sum_{i=1}^{k} p_{i j} \Delta\left(\beta_{i}\right)=2^{n+1}, 2^{n}, 0$ and hence

$$
\sum_{i=1}^{k} p_{i j} \Delta\left(\beta_{i}\right)=2^{n}, 0,-2^{n}, j=0,1, \ldots, 2^{n}-1
$$

For each fixed $j$, rewrite 6 as

$$
p_{1 j} \Delta\left(\beta_{1}\right)+\sum_{i=2}^{k} p_{i j} \Delta\left(\beta_{i}\right)=2^{n}, 0,-2^{n}
$$

By using Lemma 3 there exists a number $j_{1}, 0 \leq j_{1} \leq 2^{n-1}-1$, such that $\left(p_{1 j_{1}}, p_{2 j_{1}}, \ldots, p_{k j_{i}}\right)=\left(p_{1 j},-p_{2 j}, \ldots,-p_{k j}\right)$. 
Hence

$$
p_{1 j_{1}} \Delta\left(\beta_{1}\right)-\sum_{i=2}^{k} p_{i j_{1}} \Delta\left(\beta_{i}\right)=2^{n}, 0,-2^{n}
$$

Adding 7 and 8 together, we have

$$
p_{1 j} \Delta\left(\beta_{1}\right)= \pm 2^{n}, \pm 2^{n-1}, 0
$$

Since $\Delta\left(\beta_{1}\right) \neq 0$, we conclude $\Delta\left(\beta_{1}\right)= \pm 2^{n}, \pm 2^{n-1}$. By the same reasoning we can prove

$$
\Delta\left(\beta_{j}\right)= \pm 2^{n}, \pm 2^{n-1}, j=1,2, \ldots, k
$$

Thus we can write

$$
\left(\Delta\left(\beta_{1}\right), \ldots, \Delta\left(\beta_{k}\right)\right)=2^{n-1}\left(b_{1}, \ldots, b_{k}\right)
$$

where each $b_{j}= \pm 1, \pm 2$. By using Lemma 3 there exists a number $s, 0 \leq s \leq$ $2^{n-1}-1$, such that

$$
\left(p_{1 s}, \ldots, p_{k s}\right)=\left(\frac{b_{1}}{\left|b_{1}\right|}, \ldots, \frac{b_{k}}{\left|b_{j}\right|}\right) .
$$

Due to IUI and III,

$$
\sum_{i=1}^{k} p_{i s} \Delta\left(\beta_{i}\right)=\sum_{i=1}^{k} \frac{b_{i}}{\left|b_{i}\right|} \Delta\left(\beta_{i}\right)=\sum_{i=1}^{k} \frac{b_{i}^{2}}{\left|b_{i}\right|} 2^{n-1}=2^{n-1} \sum_{i=1}^{k}\left|b_{i}\right| \geq k 2^{n-1} .
$$

Since $k \geq 3, \boldsymbol{\Pi}$ contradicts $\boldsymbol{b}$.

Case 2: $n$ is even. By using Lemma 1

$$
\begin{aligned}
& \left\{a_{j}^{2}, a_{2 j+1}^{2}, a_{j_{0}}^{2}, a_{2 j_{0}+1}^{2}\right\}=\left\{2^{n+2}, 0,0,0\right\} \text { or } \\
& \left\{a_{j}^{2}, a_{2 j+1}^{2}, a_{j_{0}}^{2}, a_{2 j_{0}+1}^{2}\right\}=\left\{2^{n}, 2^{n}, 2^{n}, 2^{n}\right\}, j=0,1, \ldots, 2^{n-1}
\end{aligned}
$$

Hence from $\left[\mathbf{B}\right.$, we have $\Delta(0)+\sum_{i=1}^{k} p_{i j} \Delta\left(\beta_{i}\right)=2^{n+1}, 2^{n}, 0$, and hence

$$
\sum_{i=1}^{k} p_{i j} \Delta\left(\beta_{i}\right)=2^{n}, 0,-2^{n}
$$

Repeating the same deduction as in Case 1, we obtain a contradiction in Case 2.

Summarizing Cases 1 and 2, we conclude that the assumption that $\beta_{1}, \ldots, \beta_{k}$ are linearly independent is wrong. This proves the theorem.

Theorem 3 shows that $\Re$ is subject to crucial restrictions. We now compare Theorem 31 with Theorem 2 Since $n+1$ non-zero vectors in $V_{n}$ must be linearly dependent, Theorem $\mathbb{Z}$ is trivial when $\# \Re \geq n+2$ (i.e., $\#(\Re-\{0\}) \geq n+1)$. In contrast, in Theorem $\mathbf{3}$ the linear dependence of vectors takes place in each $\Re \cap W$ not only in $\Re$.

We notice that there exist $n-1(n-1)$-dimensional linear subspaces. Hence Theorem $\mathbf{3}$ is more profound than Theorem $\mathbf{Z}$ 


\section{The Unbiased Distribution of $\Re$}

In this section we focus on the distribution of $\Re$ for the functions on $V_{n}$, whose nonlinearity does not take the special value $2^{n-1}-2^{\frac{1}{2}(n-1)}$ or $2^{n-1}-2^{\frac{1}{2} n}$ or $2^{n-1}-2^{\frac{1}{2} n-1}$.

The next result is from 5 (Theorem 18).

Lemma 6. Let $f$ be a function on $V_{n}(n \geq 2)$, $\xi$ be the sequence of $f$, and $p$ is an integer, $2 \leq p \leq n$. If $\left\langle\xi, \ell_{j}\right\rangle \equiv 0 \quad\left(\bmod 2^{n-p+2}\right)$, where $\ell_{j}$ is the $j$ th row of $H_{n}, j=0,1, \ldots, 2^{n}-1$, then the algebraic degree of $f$ is at most $p-1$.

Lemma 7. For every function $f$ on $V_{n}$, we have

$$
\begin{aligned}
& 4\left(\Delta\left(\alpha_{0}\right), \Delta\left(\alpha_{4}\right), \ldots, \Delta\left(\alpha_{2^{n}-4}\right)\right) H_{n-2} \\
& =\left(\sum_{j=0}^{3}\left\langle\xi, \ell_{j}\right\rangle^{2}, \sum_{j=4}^{7}\left\langle\xi, \ell_{j}\right\rangle^{2}, \ldots, \sum_{j=2^{n}-4}^{2^{n}-1}\left\langle\xi, \ell_{j}\right\rangle^{2}\right)
\end{aligned}
$$

Where $\xi$ denotes the sequence of $f$ and $\ell_{i}$ is the ith row of $H_{n}, i=0,1, \ldots, 2^{n}-1$.

Proof. Comparing the $4 j$ th terms, $j=0,1, \ldots, 2^{n-2}-1$, in the two sides of equality II), we obtain

$$
\begin{aligned}
& 2^{n}\left(\Delta\left(\alpha_{0}\right), \Delta\left(\alpha_{4}\right), \ldots, \Delta\left(\alpha_{2^{n}-4}\right)\right) \\
& =\left(\sum_{j=0}^{3}\left\langle\xi, \ell_{j}\right\rangle^{2}, \sum_{j=4}^{7}\left\langle\xi, \ell_{j}\right\rangle^{2}, \ldots, \sum_{j=2^{n}-4}^{2^{n}-1}\left\langle\xi, \ell_{j}\right\rangle^{2}\right) H_{n-2}
\end{aligned}
$$

This proves the lemma.

Theorem 4. Let $f$ be a function on $V_{n}$, and $U$ be a $(n-2)$-dimensional linear subspace satisfying $\#(\Re \cap U)=1$ (i.e., $\Re \cap U=\{0\})$. Then we have

(i) if $n$ is odd, then the nonlinearity of $f$ satisfies $N_{f}=2^{n-1}-2^{\frac{1}{2}(n-1)}$ and the algebraic degree of $f$ is at most $2^{\frac{1}{2}(n+1)}$,

(ii) if $n$ is even, then $f$ is bent or the nonlinearity of $f$ satisfies $N_{f}=2^{n-1}-2^{\frac{1}{2} n}$ and the algebraic degree of $f$ is at most $2^{\frac{1}{2} n+1}$.

Proof. We only need to prove the theorem in the special case when $U$ is composed of $\alpha_{0}, \alpha_{4}, \alpha_{8}, \ldots, \alpha_{2^{n}-4}$, where $\alpha_{4 j} \in V_{n}$ is the binary representation of even number $4 j, j=0,1,2, \ldots, 2^{n-2}-1$. In other words, $U$ is composed of all the vectors in $V_{n}$, that can be expressed in the form $\left(a_{1}, \ldots, a_{n-2}, 0,0\right)$, where each $a_{j} \in G F(2)$. For $U$ in general case, we can use a nonsingular linear transformation on the variables so as to change $U$ into the special case. Let $\xi$ be the sequence of $f$. Set $a_{j}^{2}=\left\langle\xi, \ell_{j}\right\rangle^{2}, j=0,1, \ldots, 2^{n}-1$. 
Since $\Delta(0)=2^{n}$ and $\Delta\left(\alpha_{4 j}\right)=0, j=1,2, \ldots, 2^{n-2}-1$, the equality in Lemma $\mathbf{7}$ is specialized as

$$
2^{n+2}(1, \ldots, 1)=\left(\sum_{j=0}^{3} a_{j}^{2}, \sum_{j=4}^{7} a_{j}^{2}, \ldots, \sum_{j=2^{n}-4}^{2^{n}-1} a_{j}^{2}\right)
$$

$j=0,1, \ldots, 2^{n-2}-1$.

(i) When $n$ is odd, by using Lemma 4

$$
\left\{a_{4 j}^{2}, a_{4 j+1}^{2}, a_{4 j+3}^{2}, a_{4 j+3}^{2}\right\}=\left\{2^{n+1}, 2^{n+1}, 0,0\right\}, j=0,1, \ldots, 2^{n-2}
$$

By using Lemma II we have proved the nonlinearity of $f$ satisfies $N_{f}=$ $2^{n-1}-2^{\frac{1}{2}(n-1)}$, and by using Lemma 6 we have proved that the algebraic degree of $f$ is at most $2^{\frac{1}{2}(n+1)}$.

(ii) When $n$ is even. By using Lemma 1

$$
\left\{a_{4 j}^{2}, a_{4 j+1}^{2}, a_{4 j+3}^{2}, a_{4 j+3}^{2}\right\}=\left\{2^{n}, 2^{n}, 2^{n}, 2^{n}\right\} \text { or }\left\{2^{n+2}, 0,0,0\right\},
$$

$j=0,1, \ldots, 2^{n-2}-1$.

If there exists a number $j_{0}, 0 \leq j_{0} \leq 2^{n-2}-1$, such that

$$
\left\{a_{4 j_{0}}^{2}, a_{4 j_{0}+1}^{2}, a_{4 j_{0}+2}^{2}, a_{4 j_{0}+3}^{2}\right\}=\left\{2^{n+2}, 0,0,0\right\}
$$

then by using Lemma II we have proved that the nonlinearity of $f$ satisfies $N_{f}=2^{n-1}-2^{\frac{1}{2} n}$, and by using Lemma 6 we have proved that the algebraic degree of $f$ is at most $2^{\frac{1}{2}(n+1)}$.

If there exists no such $j_{0}$, mentioned as above, i.e., $\left\{a_{4 j}^{2}, a_{4 j+1}^{2}, a_{4 j+3}^{2}, a_{4 j+3}^{2}\right\}=$ $\left\{2^{n}, 2^{n}, 2^{n}, 2^{n}\right\}, j=0,1, \ldots, 2^{n-2}-1$. Then $f$ is bent.

To emphasise the distribution of $\Re$ we modify Theorem 4 as follows:

Theorem 5. Let $f$ be a function on $V_{n}$. If the nonlinearity of $f$ does not take the special value $2^{n-1}-2^{\frac{1}{2}(n-1)}$ or $2^{n-1}-2^{\frac{1}{2} n}$ or $2^{n-1}-2^{\frac{1}{2} n-1}$, then $\#(\Re \cap U) \geq 2$ where $U$ is any $(n-2)$-dimensional linear subspace, in other words, every $(n-2)$ dimensional linear subspace $U$ contains a non-zero vector in $\Re$.

There exist many methods to locate all the $(n-1)$-dimensional linear subspaces and all the $(n-2)$-dimensional linear subspaces in $V_{n}$. For example, let $\varphi_{\alpha}$ denote the linear function on $V_{n}$, where $\alpha \in V_{n}$, such that $\varphi_{\alpha}(x)=\langle\alpha, x\rangle$. Hence $W=\left\{\gamma \mid \alpha \in V_{n}, \varphi_{\alpha}(\gamma)=0\right\}$ is a $(n-1)$-dimensional linear subspace and each $(n-1)$-dimensional linear subspace can be expressed in this form.

Also for any $\alpha, \alpha^{\prime} \in V_{n}$ with $\alpha \neq \alpha^{\prime}, U=\left\{\gamma \mid \alpha \in V_{n}, \varphi_{\alpha}(\gamma)=0, \varphi_{\alpha^{\prime}}(\gamma)=0\right\}$ is a $(n-2)$-dimensional linear subspace and each $(n-2)$-dimensional linear subspace can be expressed in this form.

Lemma 8. Let $\Omega$ be a subset of $V_{k}$ with $0 \notin \Omega$. If there exists a positive integer $p$ such that $\#(\Omega \cap U) \geq p$ holds for every $(k-1)$-dimensional linear subspace $U$, then $\# \Omega \geq 2 p+1$. 
Proof. Note that each non-zero vector is included in precisely $2^{k-1}-1(k-1)$ dimensional linear subspaces, on the other hand, there exist exactly $2^{k}-1(k-$ $1)$-dimensional linear subspaces. Hence $\left(2^{k-1}-1\right) \# \Omega=\sum_{U} \#(\Omega \cap U)$. From $\#(\Omega \cap U) \geq p$, we conclude that $\left(2^{k-1}-1\right) \# \Omega \geq\left(2^{k}-1\right) p$. Since $\frac{2^{k}-1}{2^{k-1}-1}>2$, $\# \Omega>2 p$ or $\# \Omega \geq 2 p+1$.

Theorem 6. Let $f$ be a function on $V_{n}$. If the nonlinearity of $f$ does not take the special values $2^{n-1}-2^{\frac{1}{2}(n-1)}$ or $2^{n-1}-2^{\frac{1}{2} n}$ or $2^{n-1}-2^{\frac{1}{2} n-1}$, then $\#(\Re \cap W) \geq 4$ for every $(n-1)$-dimensional linear subspace $W$, in other words, every $(n-1)$ dimensional linear subspace $W$ contains at least three non-zero vectors in $\Re$.

Proof. Let $W$ be an arbitrary $(n-1)$-dimensional linear subspace and $U$ be an arbitrary $(n-2)$-dimensional linear subspace with $U \subset W$. Note that the inequality in Theorem $\mathbf{n}$ can be rewritten as

$$
\#((\Re-\{0\}) \cap U) \geq 1
$$

and $((\Re-\{0\}) \cap W) \cap U=(\Re-\{0\}) \cap U$. Applying Lemma 8 we have proved $\#((\Re-\{0\}) \cap W) \geq 3$. Since $0 \in \Re \cap W, \#(\Re \cap W) \geq 4$.

Theorems 5 and 6 are helpful to locate the non-propagative vectors.

The properties mentioned together in Theorems 5 and 6 are called the unbiased distribution of $\Re$, with respect to every $(n-2)$-dimensional linear subspace and every $(n-1)$-dimensional linear subspace.

\section{$6 \quad$ Distribution of $\Re$ in Special Cases}

We now turn to the case \# $\left(\Re_{f} \cap W\right) \leq 3$ where $W$ is an $(n-1)$-dimensional linear subspace. The following Lemma can be found in $\mathbf{7}$ :

Lemma 9. Let $n \geq 2$ be a positive integer and $2^{n}=a^{2}+b^{2}$ where $a \geq b \geq 0$ and both $a$ and $b$ are integers. Then $a^{2}=2^{n}$ and $b=0$ when $n$ is even, and $a^{2}=b^{2}=2^{n-1}$ when $n$ is odd.

Theorem 7. Let $f$ be a function on $V_{n}$, and $W$ be an $(n-1)$-dimensional linear subspace satisfying $\#(\Re \cap W)=1$ (i.e., $\Re \cap W=\{0\})$. We have

(i) $f$ has at most one non-zero linear structure,

(ii) if $n$ is odd, then the nonlinearity of $f$ satisfies $N_{f}=2^{n-1}-2^{\frac{1}{2}(n-1)}$ and the algebraic degree of $f$ is at most $2^{\frac{1}{2}(n+1)}$,

(iii) if $n$ is even, then $f$ is bent.

Proof. (i) Let $\alpha^{*} \in V_{n}$ and $\alpha^{*} \notin W$, From linear algebra, $V_{n}=W \cup\left(\alpha^{*} \oplus W\right)$, where $\alpha^{*} \oplus W=\left\{\alpha^{*} \oplus \alpha \mid \alpha \in W\right\}, W$ and $\alpha^{*} \oplus W$ are disjoint. We now prove that $f$ has at most one non-zero linear structure by contradiction. Suppose $f$ has two 
non-zero linear structures, $\beta_{1}$ and $\beta_{2}$ with $\beta_{1} \neq \beta_{2}$. Since all linear structures of $f$ form a linear subspace of $V_{n}, \beta_{1} \oplus \beta_{2}$ is also a non-zero linear structures of $f$ and hence $\beta_{1} \oplus \beta_{2} \in \Re$. Since $\Re \cap W=\{0\}, \beta_{1}, \beta_{2} \in \alpha^{*} \oplus W$. Obviously $\beta_{1} \oplus \beta_{2} \in W$ and hence $\beta_{1} \oplus \beta_{2} \in \Re \cap W$. This contradicts the condition $\Re \cap W=\{0\}$. The contradiction proves that $f$ has at most one non-zero linear structure.

Recall the proof of Theorem 3 B can be specialized as $2 \Delta(0)=a_{2 j}^{2}+a_{2 j+1}^{2}$ and hence $a_{2 j}^{2}+a_{2 j+1}^{2}=2^{n+1}$, where $j=0,1, \ldots, 2^{n-1}-1$.

(ii) If $n$ be odd, from Lemma $\mathbf{g}\left\{a_{2 j}^{2}, a_{2 j+1}^{2}\right\}=\left\{2^{n+1}, 0\right\}$, where $j=0,1, \ldots$, $2^{n-1}-1$. From Lemma 11 the nonlinearity of $f$ satisfies $N_{f}=2^{n-1}-2^{\frac{1}{2}(n-1)}$. By using Lemma 6 we conclude that the algebraic degree of $f$ is at most $2^{\frac{1}{2}(n+1)}$.

(iii) If $n$ is even, due to Lemma $9 a_{2 j}^{2}=a_{2 j+1}^{2}=2^{n}$, where $j=0,1, \ldots, 2^{n-1}-$ 1. This proves that $f$ is bent.

Example 1. Let $n$ be a positive odd number and $f\left(x_{1}, \ldots\right.$, $\left.x_{n}\right)=x_{1} \oplus g\left(x_{2}, \ldots, x_{n}\right)$ where $g$ is a bent function in $V_{n-1}$. Let $W$ be an $(n-1)$-dimensional linear subspace of $V_{n}$, composed of all the vectors in $V_{n}$, that can be expressed in the form $\left(0, a_{2}, \ldots, a_{n}\right)$, where each $a_{j} \in G F(2)$. It is easy to see $\alpha^{*}=(1,0, \ldots, 0) \in V_{n}$ is a non-zero linear structure of $f$ and $\Re \cap W=\{0\}$. Due to (ii) of Theorem $\mathbf{n} N_{f}=2^{n-1}-2^{\frac{1}{2}(n-1)}$.

We can restate (iii) of Theorem $\boldsymbol{\nabla}$ as follows:

Proposition 1. Let $f$ be a function on $V_{n}$ where $n$ is even. If there exists an $(n-1)$-dimensional linear subspace $W_{0}$ satisfying $\#\left(\Re \cap W_{0}\right)=1$ (i.e., $\Re \cap W_{0}=$ $\{0\})$, then $f$ satisfies $\Re \cap W=\{0\}$, for every $(n-1)$-dimensional linear subspace $W$.

Next we examine the case of $\#(\Re \cap W)=2$.

Theorem 8. Let $f$ be a function on $V_{n}$. If there exists a $(n-1)$-dimensional linear subspace $W$ satisfying $\Re \cap W=\left\{0, \beta_{1}\right\}$, then we have

(i) $\beta_{1}$ is a non-zero linear structure of $f$,

(ii) if $n$ is odd, then the nonlinearity of $f$ satisfies $N_{f}=2^{n-1}-2^{\frac{1}{2}(n-1)}$ and the algebraic degree of $f$ is at most $2^{\frac{1}{2}(n+1)}$,

(iii) if $n$ is even, then $N_{f}=2^{n-1}-2^{\frac{1}{2} n}$ and the algebraic degree of $f$ is at most $2^{\frac{1}{2} n+1}$.

Proof. Since any single non-zero vector is linearly independent, we can keep the deduction in the proof of Theorem $\mathbf{3}$ until inequality $\mathbf{F}$ where we need the condition $k \geq 3$.

(i) Recall the proof of Theorem 36 can be specialized as $p_{1 j} \Delta\left(\beta_{1}\right)=$ $2^{n}, 0,-2^{n}, j=0,1, \ldots, 2^{n}-1$. Since $\beta_{1} \in \Re, \Delta\left(\beta_{1}\right) \neq 0$. Hence $\Delta\left(\beta_{1}\right)= \pm 2^{n}$. This proves that $\beta_{1}$ is a non-zero linear structure. 
(ii) If $n$ is odd, from 5 we conclude that $\left\langle\xi, \ell_{i}\right\rangle^{2}=2^{n+1}, 0, i=0,1, \ldots, 2^{n}-1$, and hence by using Lemma II we have proved $N_{f}=2^{n-1}-2^{\frac{1}{2}(n-1)}$. By using Lemma 6 we conclude that the algebraic degree of $f$ is at most $2^{\frac{1}{2}(n+1)}$.

(iii) If $n$ is even, from [I.3, $\left\langle\xi, \ell_{i}\right\rangle^{2}=2^{n+2}, 0,2^{n}$. Since $\# \Re>1, f$ is not bent. Hence $\left\langle\xi, \ell_{i}\right\rangle^{2}=2^{n}$ cannot hold for all $i$ and hence there exists a number $i_{0}, 0 \leq i_{0} \leq 2^{n}-1$, such that $\left\langle\xi, \ell_{i}\right\rangle^{2}=2^{n+2}$. By using Lemma II we have proved $N_{f}=2^{n-1}-2^{\frac{1}{2} n}$, if $n$ is even. By using Lemma $\mathbf{0}$ we conclude that the algebraic degree of $f$ is at most $2^{\frac{1}{2} n+1}$.

Example 2. Let $n$ be a positive odd number and $f\left(x_{1}, \ldots, x_{n}\right)$ be the same with that in Example II Let $W$ be an $(n-1)$-dimensional linear subspace of $V_{n}$, composed of all the vectors in $V_{n}$, that can be expressed in the form $\left(a_{1}, \ldots, a_{n-1}, 0\right)$, where each $a_{j} \in G F(2)$. It is easy to see $\alpha^{*}=(1,0, \ldots, 0) \in V_{n}$ is a nonzero linear structure of $f$ and $\Re \cap W=\left\{0, \alpha^{*}\right\}$. Due to (ii) of Theorem $\&$ $N_{f}=2^{n-1}-2^{\frac{1}{2}(n-1)}$.

Let $k$ be a positive even number with $k \geq 4$ and $h\left(x_{1}, \ldots, x_{k}\right)=x_{1} \oplus$ $x_{2} \oplus q\left(x_{3}, \ldots, x_{k}\right)$ where $q$ is a bent function on $V_{k-2}$. Let $U$ be an $(n-1)$ dimensional linear subspace of $V_{n}$, composed of all the vectors in $V_{n}$, that can be expressed in the form $\left(0, a_{2}, \ldots, a_{k}\right)$, where each $a_{j} \in G F(2)$. It is easy to see $\alpha_{1}^{*}=(0,1,0, \ldots, 0)$ is a non-zero linear structures of $h$ and $\Re \cap U=\left\{0, \alpha_{1}^{*}\right\}$. Due to (iii) of Theorem $8 N_{h}=2^{k-1}-2^{\frac{1}{2} k}$.

It is interesting that by using Theorem 8 we have determined $N_{h}$ only from the condition $\#(\Re \cap U)=2$ for an $(n-1)$-dimensional linear subspace $U$ although we do not search other vectors in $\Re$.

Finally, we consider the case when $\#(\Re \cap W)=3$.

Theorem 9. Let $f$ be a function on $V_{n}$. If there exists a $(n-1)$-dimensional linear subspace $W$ satisfying $\Re \cap W=\left\{0, \beta_{1}, \beta_{2}\right\}$, then the following statements hold:

(i) $\Delta\left(\beta_{j}\right)= \pm 2^{n-1}, j=1,2$,

(ii) if $n$ is odd, then the nonlinearity of $f$ satisfies $N_{f}=2^{n-1}-2^{\frac{1}{2}(n-1)}$ and the algebraic degree of $f$ is at most $2^{\frac{1}{2}(n+1)}$,

(iii) if $n$ is even, then $N_{f}=2^{n-1}-2^{\frac{1}{2} n}$ and the algebraic degree of $f$ is at most $2^{\frac{1}{2} n+1}$.

Proof. Since any two non-zero vectors are linearly independent, we can keep the deduction in the proof of Theorem 3 until inequality 12 where we need the condition $k \geq 3$.

Recall the proof of Theorem 39 can be specialized as $\Delta\left(\beta_{j}\right)= \pm 2^{n}, \pm 2^{n-1}$, $j=1,2$.

On the other hand, IIU, III and ID can be rewritten as $\left(\Delta\left(\beta_{1}\right), \Delta\left(\beta_{2}\right)\right)=$ $2^{n-1}\left(b_{1}, b_{2}\right)$ where each $b_{j}= \pm 1, \pm 2,\left(p_{1 s}, p_{2 s}\right)=\left(\frac{b_{1}}{\left|b_{1}\right|}, \frac{b_{2}}{\left|b_{2}\right|}\right)$. and

$$
p_{1 s} \Delta\left(\beta_{1}\right)+p_{2 s} \Delta\left(\beta_{2}\right)=\left(\left|b_{1}\right|+\left|b_{2}\right|\right) 2^{n-1}
$$


respectively. It is easy to prove $b_{1}, b_{2}= \pm 1$. Otherwise, for example, $b_{1}= \pm 2$, from 16, $p_{1 s} \Delta\left(\beta_{1}\right)+p_{2 s} \Delta\left(\beta_{2}\right) \geq 3 \cdot 2^{n-1}$. This contradicts 6 . Since $b_{1}, b_{2}= \pm 1$, $\Delta\left(\beta_{1}\right), \Delta\left(\beta_{2}\right)= \pm 2^{n-1}$. This proves (i).

The rest proof is the same with the proof of Theorem 8

Example 3. Let $n$ be a positive odd number with $n \geq 7, h\left(x_{1}, x_{2}, x_{3}, x_{4}, x_{5}\right)=$ $\left(x_{1} \oplus x_{2} \oplus x_{3}\right) x_{4} x_{5} \oplus x_{1} x_{5} \oplus x_{2} x_{4} \oplus x_{1} \oplus x_{2} \oplus x_{3}$ and $g\left(x_{6}, \ldots, x_{n}\right)$ be a bent function on $V_{n-5}$. Set $f\left(x_{1}, \ldots, x_{n}\right)=h\left(x_{1}, x_{2}, x_{3}, x_{4}, x_{5}\right) \oplus g\left(x_{6}, \ldots, x_{n}\right)$.

Let $W$ be an $(n-1)$-dimensional linear subspace of $V_{n}$, composed of all the vectors in $V_{n}$, that can be expressed in the form $\left(0, a_{2}, \ldots, a_{n}\right)$, where each $a_{j} \in G F(2)$. Write $\alpha_{1}^{*}=(0,0,1,0, \ldots, 0), \alpha_{2}^{*}=(0,1,0, \ldots, 0) \in V_{n}$, It is easy to verify $\alpha_{1}^{*}, \alpha_{2}^{*} \in \Re$ and $\Re \cap W=\left\{0, \alpha_{1}^{*}, \alpha_{2}^{*}\right\}$. Due to (i) and (ii) of Theorem 9 we conclude $\Delta\left(\alpha_{1}^{*}\right)= \pm 2^{n-1}, \Delta\left(\alpha_{2}^{*}\right)= \pm 2^{n-1}$ and $N_{f}=2^{n-1}-2^{\frac{1}{2}(n-1)}$.

We notice that by using Theorem 9 we have determined $N_{h}, \Delta\left(\alpha_{1}^{*}\right)$ and $\Delta\left(\alpha_{2}^{*}\right)$ only from the information about $\#(\Re \cap W)$ for an $(n-1)$-dimensional linear subspace $W$ although we do not search other the vectors in $\Re$.

We can also find an example corresponding to (iii) of Theorem 9 All Theorems $\mathbf{7} \quad \mathbf{8}$ and $\mathbf{9}$ and Examples $\mathbf{\|} \quad \mathbf{2}$ and $\mathbf{3}$ show that we can determine the nonlinearity of a function only from some information about $\#(\Re \cap W)$, where $W$ is an $(n-1)$-dimensional linear subspace. It is interesting that 7 has proved that there exists no a function with $\# \Re=3$ while Example 3 gives a function satisfying $\#(\Re \cap W)=3$ for an $(n-1)$-dimensional linear subspace $W$.

\section{Conclusions}

The strong linear dependence is an improvement on a previously known result. The unbiased distribution of non-propagation vectors is valid for most functions. These results provide more information on the non-propagative vectors in any $(n-1)$-dimensional linear subspace of $V_{n}$, and hence they are helpful for designing cryptographic functions.

\section{Acknowledgement}

The second author was supported by a Queen Elizabeth II Fellowship (227 23 1002).

\section{References}

1. Claude Carlet. Partially-bent functions. Designs, Codes and Cryptography, 3:135145, 1993.

2. W. Meier and O. Staffelbach. Nonlinearity criteria for cryptographic functions. In Advances in Cryptology - EUROCRYPT'89, volume 434, Lecture Notes in Computer Science, pages 549-562. Springer-Verlag, Berlin, Heidelberg, New York, 1990. 
3. B. Preneel, W. V. Leekwijck, L. V. Linden, R. Govaerts, and J. Vandewalle. Propagation characteristics of boolean functions. In Advances in Cryptology - EUROCRYPT'90, volume 437, Lecture Notes in Computer Science, pages 155-165. Springer-Verlag, Berlin, Heidelberg, New York, 1991.

4. O. S. Rothaus. On "bent" functions. Journal of Combinatorial Theory, Ser. A, 20:300-305, 1976.

5. A. F. Webster and S. E. Tavares. On the design of S-boxes. In Advances in Cryptology - CRYPTO'85, volume 219, Lecture Notes in Computer Science, pages 523-534. Springer-Verlag, Berlin, Heidelberg, New York, 1986.

6. Y. Zheng X. M. Zhang and Hideki Imai. Duality of boolean functions and its cryptographic significance. In Advances in Cryptology - ICICS'97, volume 1334, Lecture Notes in Computer Science, pages 159-169. Springer-Verlag, Berlin, Heidelberg, New York, 1997.

7. X. M. Zhang and Y. Zheng. Characterizing the structures of cryptographic functions satisfying the propagation criterion for almost all vectors. Design, Codes and Cryptography, 7(1/2):111-134, 1996. special issue dedicated to Gus Simmons. 